\title{
DNA photodamage: Study of cyclobutane pyrimidine dimer formation in a locked thymine dinucleotide
}

\author{
W.J. Schreier ${ }^{\text {a }}$, J. Kubon ${ }^{\text {a }}$, P. Clivio ${ }^{\text {b }}$, W. Zinth ${ }^{\text {a,* }}$ and P. Gilch ${ }^{\mathrm{a}, * *}$ \\ ${ }^{a}$ Fakultät für Physik, LMU München, Lehrstuhl für BioMolekulare Optik, München, Germany and \\ Munich Center for Integrated Protein Science CIPSM, München, Germany \\ ${ }^{\mathrm{b}}$ Institut de Chimie Moléculaire de Reims, Université de Reims Champagne Ardenne, Reims, France
}

\begin{abstract}
The cyclobutane pyrimidine dimer (CPD) formed between two adjacent thymine bases is the most abundant DNA photolesion induced by UV radiation. The quantum yield of this reaction is on the order of $\sim 1 \%$ in DNA. This small quantum yield hampers the study of damage formation in naturally occurring DNA. Investigations with increased accuracy become possible for a locked nucleotide model compound $\mathrm{T}_{\mathrm{L}} \mathrm{pT} \mathrm{T}_{\mathrm{L}}$ which exhibits a quantum yield of about $10 \%$ for CPD formation. Time resolved IR spectroscopy on $\mathrm{T}_{\mathrm{L}} \mathrm{TT}_{\mathrm{L}}$ and two other DNA model compounds (TpT and (dT) 18 ) reveals that: (i) The absorption changes after $\sim 1$ ps are due to CPD photodamage. (ii) The quantum efficiency of CPD formation on the few picosecond time scale equals the quantum efficiency reported in stationary experiments. CPD photodamage formation in the investigated DNA constructs is thus predominantly formed from the primarily photoexcited singlet $\pi \pi^{*}$ state, whereas the triplet channel does not play an essential role.
\end{abstract}

Keywords: Infrared spectroscopy, DNA photolesion, CPD damage, thymine dimer

\section{Introduction}

UV radiation is known to be responsible for a variety of photo lesions in DNA. It is one of the most important external hazards for the integrity of the genetic information and may lead to skin cancer [24]. The most abundant photo lesion is due to the formation of the cyclobutane pyrimidine dimer (CPD) between two thymines adjacent on a DNA strand [25]. The quantum yield of this reaction was found to be on the order of $\sim 1 \%$ for DNA [8,10]. Although described as early as 1960 [2,3], there is still an ongoing debate on the reaction mechanisms leading to CPD formation in DNA.

Irradiation experiments on single thymine bases in diluted solutions demonstrate that CPD's can be formed in a diffusion limited process via a long lived triplet state [11]. Nevertheless this process must not

\footnotetext{
${ }^{*}$ Corresponding author: W. Zinth, Fakultät für Physik, LMU München, Lehrstuhl für BioMolekulare Optik, Oettingen str. 67, 80538 München, Germany and Munich Center for Integrated Protein Science CIPSM, München, Germany. Tel.: +49 892180 9200; Fax: +49 892180 9202; E-mail: wolfgang.zinth@physik.uni-muenchen.de.

*** Present address: Institut für Physikalische Chemie, Heinrich-Heine-Universität Düsseldorf Universitäts str. 1, 40225 Düsseldorf, Germany.
} 
play an important role for CPD formation in a DNA strand where the bases are hold in close proximity by the sugar-phosphate backbone. In this case CPD-formation could also occur via a short lived singlet state [9].

To elucidate the ultrafast reaction mechanisms one needs a technique with high temporal resolution and spectral markers that allow for an unambiguous assignment of the damage formation. As the formation of CPD lesions does not produce distinct spectral marker bands in the UV/Vis range we performed a time resolved pump probe experiment with UV excitation and probing in the mid-IR region, where characteristic marker bands for dimer formation are present. With this technique we have recently traced the formation of CPD lesions in an all thymine DNA single strand $\left((\mathrm{dT})_{18}\right)$ and demonstrated that marker bands for CPD formation are present as early as $1 \mathrm{ps}$ after UV-excitation [23]. This finding points to CPD formation from the singlet $\pi \pi^{*}$ state since excited electronic states other than the primarily excited singlet $\pi \pi^{*}$ state are much longer lived [18].

Simulations have shown that large-scale conformational changes in DNA single strands [18] do not occur on the time scale of a few picoseconds. Therefore the DNA strand can be assumed to be essentially static during the 1 ps dimerisation reaction. Based on this we have postulated [23] that the small value of the quantum yield $\phi_{\mathrm{CPD}}$ for CPD formation ( $\sim 3 \%$ value found for $\left.(\mathrm{dT})_{20}[17]\right)$ is due to the rareness of reactive conformations in a thermal ensemble.

More recently femtosecond pump probe experiment based on time resolved transient spectroscopy in the visible and UV questioned the dominance of ultrafast CPD formation and thereby the conformational control of the quantum yield [15]. For (monomeric) thymidine (dT) a spectral species with a lifetime of about $4 \mathrm{~ns}$ was found. A similar spectral signature was observed for $(\mathrm{dT})_{20}$ with a reduced lifetime of about 140 ps. The acceleration has been interpreted as reactive quenching of triplet states eventually resulting in CPD formation on the 100 ps time scale.

In all time resolved experiments the precise determination of quantum yields for different reaction channels is hampered by the small yield of damage formation. Therefore the systematic study of a series of all thymine model systems with various quantum yields and of compounds with increased yield should help to answer the question on the nature of CPD-formation. Following this idea we performed femtosecond UV pump, IR probe experiments on three dimerizable samples and a reference sample (thymidine monophosphate, TMP) [22]. The dimerizable samples and their respective quantum yields $\phi_{\mathrm{CPD}}^{\mathrm{cw}}$ (in brackets) were: the dinucleotide thymidylyl $\left(3^{\prime}-5^{\prime}\right)$ thymidine (TpT) $(1-2 \%$ [12]), the all thymine oligonucleotide $(\mathrm{dT})_{18}\left(2.8 \%\right.$ yield for the closely related $(\mathrm{dT})_{20}$ [17]), and $\mathrm{T}_{\mathrm{L}} \mathrm{pT} \mathrm{L}_{\mathrm{L}}(\sim 6$ times that of TpT [7]). In $T_{L} p T_{L}$ the furanose moieties of the deoxyriboses are forced into a $C 3^{\prime}$ endo conformation by a methylene clamp (see structure in Fig. 1(a)). This modification increases the propensity towards CPD formation by a factor of six to seven with respect to TpT. In the following we will show that in all thymine DNA models the CPD yield is settled after one picosecond and that this yield depends on the fraction of reactive conformers.

\section{Materials and methods}

\subsection{Materials}

Thymidine $5^{\prime}$-monophosphate (disodium salt hydrate) and thymidylyl $\left(3^{\prime}-5^{\prime}\right)$ thymidine (ammonium salt) were obtained from Sigma Aldrich. The modified dinucleotide $T_{L} \mathrm{pT}_{\mathrm{L}}$ was prepared and purified 

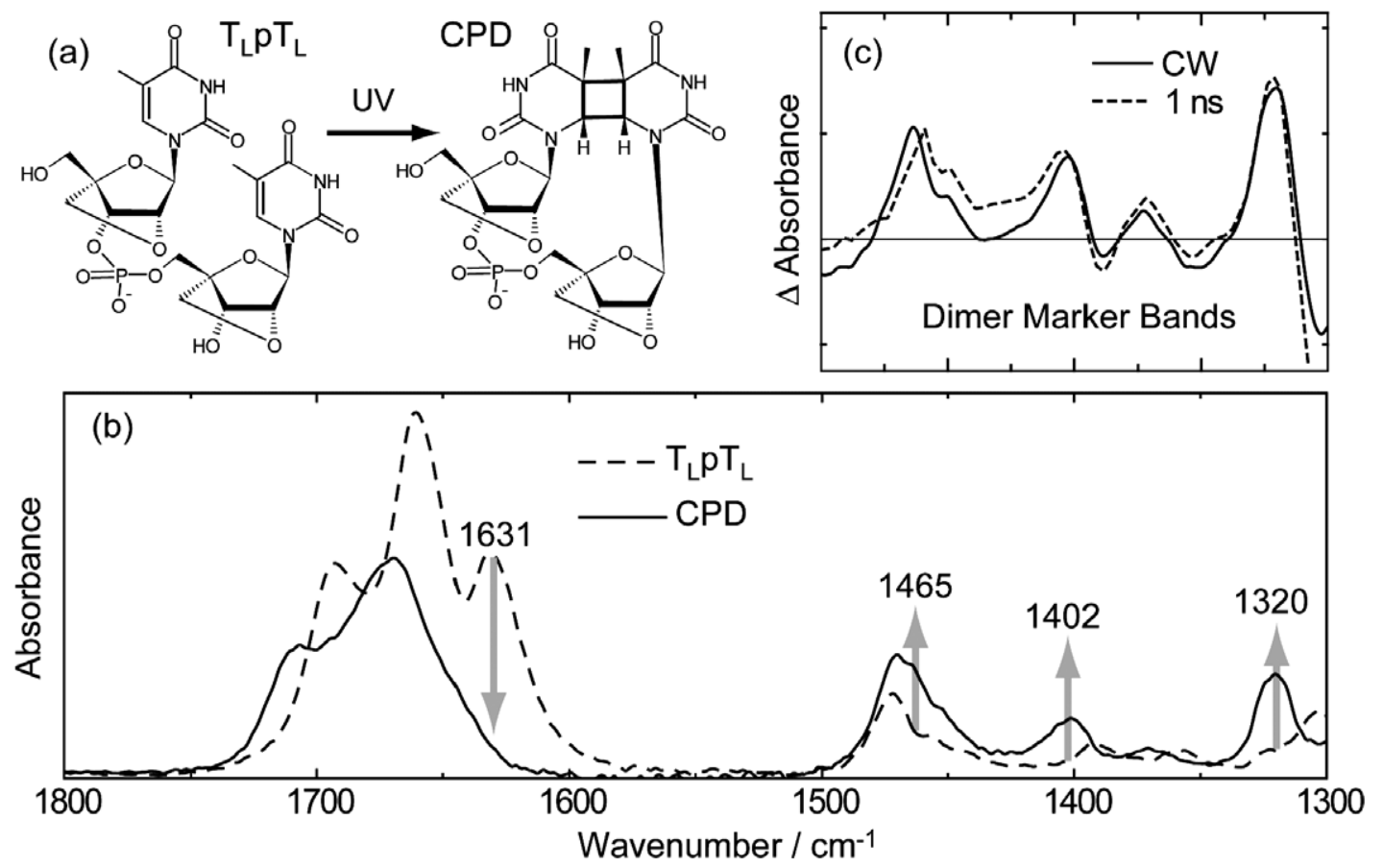

Fig. 1. (a) Chemical structures of the $\mathrm{C}^{\prime}$ endo-locked derivative of $\mathrm{TpT}\left(\mathrm{T}_{\mathrm{L}} \mathrm{pT} \mathrm{L}_{\mathrm{L}}\right)$ and the CPD lesion formed upon UV illumination. (b) IR absorption spectra of $\mathrm{T}_{\mathrm{L}} \mathrm{pT}_{\mathrm{L}}$ (dashed line) and its CPD form (solid line) in $\mathrm{D}_{2} \mathrm{O}$ buffered solution. The absorption of the $\mathrm{C}=\mathrm{C}$ double bond of the thymine base at $1631 \mathrm{~cm}^{-1}$ is missing in the $\mathrm{CPD}$ lesion. Instead there is an increase in absorption at three distinct positions in the fingerprint region below $1500 \mathrm{~cm}^{-1}$. (c) Comparison of difference spectra obtained for $\mathrm{T}_{\mathrm{L}} \mathrm{pT}_{\mathrm{L}}$ in a steady state (solid line) and fs IR experiment $1 \mathrm{~ns}$ after UV excitation (dashed line). The steady state experiment exhibits characteristic dimer marker bands for CPD formation that are also present in the time resolved measurement.

as described previously [7]. The sodium salt of the all-thymine oligonucleotide (dT) ${ }_{18}$ was purchased from Eurogentec. All samples were obtained as lyophilized powders and were dissolved in $99.9 \% \mathrm{D}_{2} \mathrm{O}$ containing $50 \mathrm{mM}$ potassium phosphate buffer. $\mathrm{D}_{2} \mathrm{O}$ instead of $\mathrm{H}_{2} \mathrm{O}$, was chosen as solvent in order to maximize IR transmittance. Concentrations per residue were determined by analysis of UV-Absorption at $260 \mathrm{~nm}$ assuming the following extinction coefficients $(\varepsilon)$ : $8.56 \times 10^{3} \mathrm{M}^{-1} \mathrm{~cm}^{-1}$ for TMP [5], $8.64 \times 10^{3} \mathrm{M}^{-1} \mathrm{~cm}^{-1}$ for TpT [12], $8.34 \times 10^{3} \mathrm{M}^{-1} \mathrm{~cm}^{-1}$ for $\mathrm{T}_{\mathrm{L}} \mathrm{pT}_{\mathrm{L}}$ (determined from the weight of the sample) and $7.8 \times 10^{3} \mathrm{M}^{-1} \mathrm{~cm}^{-1}$ for $(\mathrm{dT})_{18}$ (calculated from the values given for (dT) $)_{9}$ in [13]). Solute concentrations per nucleotide were determined to be about $9.4 \mathrm{mM}$ for TMP, $9.7 \mathrm{mM}$ for TpT and $\mathrm{T}_{\mathrm{L}} \mathrm{pT}_{\mathrm{L}}$, and $12.8 \mathrm{mM}$ for $(\mathrm{dT})_{18}$. In the applied flow cell system $(\sim 100 \mu \mathrm{m}$ pathlength) this resulted in an optical density of about 1 OD at the excitation wavelength $(268 \mathrm{~nm})$.

\subsection{Steady state spectroscopy}

Steady-state absorption spectra were recorded using a Fourier transform infrared (FTIR) spectrometer (IFS66, Bruker, Ettlingen, Germany). Infrared absorption spectra of the samples were recorded before and after irradiation with $266 \mathrm{~nm}$ pulses (fourth harmonic of a ns Nd:YAG laser (NY60, Continuum) operated at $40 \mathrm{~Hz}$ and an average intensity of $1-2 \mathrm{~mW} / \mathrm{cm}^{2}$. 


\subsection{Transient IR spectroscopy}

For details of the femtosecond transient IR spectrometer used for UV pump/IR probe experiments see $[21,23]$. In brief, the experiment is based on a femtosecond Ti:sapphire laser system with $1 \mathrm{kHz}$ repetition rate (Spitfire Pro XP, Spectra Physics). The pump pulse was obtained by third harmonic generation of the fundamental $(\sim 800 \mathrm{~nm})$ and had a center wavelength of $268 \mathrm{~nm}$. The pump pulse with an energy of $\sim 1 \mu \mathrm{J}$ was focused in the sample to a spot size (FWHM) of about $150 \mu \mathrm{m}$. The mid-IR probe pulse was generated in a two stage optical parametric amplifier in combination with difference frequency mixing of the resulting near IR pulses in a $\mathrm{AgGaS}_{2}$ crystal. It is focused to a $100 \mu \mathrm{m}$ spot size at the sample position. The probe pulse had a bandwidth of about $150 \mathrm{~cm}^{-1}$ (FWHM) and was tuned to several discrete wavelengths in order to cover the entire region of interest $\left(1300-1550 \mathrm{~cm}^{-1}\right)$ in several overlapping data acquisitions. Temporal widths of pump and probe pulses were about 350 and $150 \mathrm{fs}$, respectively. Transient absorption spectra were recorded under magic angle conditions, using a grating spectrograph equipped with a 32-element MCT detector. For a given probe center wavelength, transient spectra were recorded for pump-probe time delays up to about $3 \mathrm{~ns}$. At each value of the pump-probe delay, approximately $10^{4}$ laser pulses were averaged resulting in a detection sensitivity of $\sim 10 \mu \mathrm{OD}$ (rms).

The samples were cycled in home built flow cells $\left(\mathrm{BaF}_{2}\right.$ windows) to allow for complete sample exchange between successive laser shots. To check for sample degradation the samples were routinely characterized by recording IR and UV absorption spectra. All measurements were performed at a room temperature of about $22.5^{\circ} \mathrm{C}$.

\subsection{Circular dichroism spectroscopy}

All thymine DNA model compounds (nucleotide (TMP), dinucleotides (TpT, $\mathrm{T}_{\mathrm{L}} \mathrm{T}_{\mathrm{L}}$ ) and the oligomer $\left.(\mathrm{dT})_{18}\right)$ were dissolved in $\mathrm{D}_{2} \mathrm{O}$ with a deuterated phosphate buffer $(0.05 \mathrm{M}, \mathrm{pH}$ 7) and kept in quartz cells ( $1 \mathrm{~mm}$ path length). The concentrations (per residue) were determined by analysis of UVabsorption at $260 \mathrm{~nm}$ assuming the extinction coefficients $(\varepsilon)$ given above. The resulting concentrations were $0.83,1.1,1.2$ and $1.25 \mathrm{mM}$ for TMP, $\mathrm{TpT}, \mathrm{T}_{\mathrm{L}} \mathrm{T}_{\mathrm{L}}$ and $(\mathrm{dT})_{18}$, respectively. Measurements of CD spectra in a range of 200-400 nm with $2 \mathrm{~nm}$ intervals were performed with a commercial CD spectrometer (AVIV Biomedical, Model 410). The sample temperature was set to $20^{\circ} \mathrm{C}$. The baseline from the pure buffer was substracted and the resulting spectra converted to molar ellipticity per residue $[\theta]$.

\section{Results and discussion}

To locate IR marker bands that are indicative for CPD formation steady-state infrared absorption spectra for the $\mathrm{C}^{\prime}$ endo-locked derivative of $\mathrm{TpT}\left(\mathrm{T}_{\mathrm{L}} \mathrm{p} \mathrm{T}_{\mathrm{L}}\right)$ and the purified CPD lesion were recorded (see Fig. 1(b)). In the spectra three strong absorption bands at 1692, 1661 and $1631 \mathrm{~cm}^{-1}$ are visible. These bands arise from double-bond stretching of the two carbonyl groups and the $\mathrm{C} 5=\mathrm{C} 6$ double-bond of the thymine base respectively $[1,26]$. As expected from the molecular structure (see Fig. 1(a)) the latter absorption is missing in the CPD lesion, giving rise to a large absorption difference at this spectral position.

Characteristic absorption differences are also visible in the fingerprint region below $1500 \mathrm{~cm}^{-1}$. Here an increased absorption is apparent around 1465, 1404 and $1320 \mathrm{~cm}^{-1}$ for the CPD lesion. As the 
expected absorption changes in the double-bond region are mostly negative and strongly overlap, the three bands in the fingerprint region became the focus of the further investigations.

The results of a steady state illumination experiment with $266 \mathrm{~nm}$ excitation are shown in Fig. 1(c) (solid line). Illumination of $\mathrm{T}_{\mathrm{L}} \mathrm{pT}_{\mathrm{L}}$ results in a growing in of IR bands in the $1500-1300 \mathrm{~cm}^{-1}$ range. By comparison with the IR absorption spectra of the purified photoproduct (solid line, Fig. 1 (b)) we can verify that (i) the CPD lesion is the main photoproduct after UV illumination and that (ii) these bands are clear marker bands for CPD formation.

$\mathrm{CW}$ illumination experiments were performed for all dimerizable thymine samples. The difference spectra in the range of the marker bands for the three samples differ slightly, but they always allow an unambiguous identification of the CPD lesion. When evaluating the CW quantum yield for TpT and $\mathrm{T}_{\mathrm{L}} \mathrm{pT}_{\mathrm{L}}$ by directly comparing the slope of absorption change under $\mathrm{UV}$ illumination at $1404 \mathrm{~cm}^{-1}$ (data not shown) we found an increase by a factor of $\sim 6$. This finding is in agreement with the ratio of quantum yields given in [7].

Based on the spectroscopic information on the expected absorption changes, the formation of CPD lesions can be monitored by femtosecond UV pump/IR probe spectroscopy. In the experiment the samples were excited with UV pulses centered at $268 \mathrm{~nm}$ close to the peak of the absorption band of thymine [4]. Spectroscopic changes were then probed by mid-IR pulses in the region of the marker bands. In Fig. 2(a) the time resolved absorption changes recorded for $\mathrm{T}_{\mathrm{L}} \mathrm{pT} \mathrm{T}_{\mathrm{L}}$ are depicted. The signal features positive and negative absorption changes. There are initial absorption dynamics on a time scale of a few picoseconds. Subsequently (throughout the complete monitored time window from 5 ps up to $1 \mathrm{~ns}$ ) the absorption stays essentially constant. With reference to the measurements on (dT) ${ }_{18}$ [23] the early absorption transients (featuring sigmoidal spectral shapes) can be assigned to vibrational cooling of ground-state $\mathrm{T}_{\mathrm{L}} \mathrm{pT} \mathrm{T}_{\mathrm{L}}$ after ultrafast internal conversion [6,14]. Additionally the absorption changes agree well with the results of the CW illumination experiment in Fig. 1(b). As demonstrated in Fig. 1(c) the difference spectrum recorded $1 \mathrm{~ns}$ after time zero (dashed line) matches the $\mathrm{cw}$-difference spectrum (solid line), giving unequivocal evidence for CPD formation. The nice coincidence with the dimer marker bands can clearly

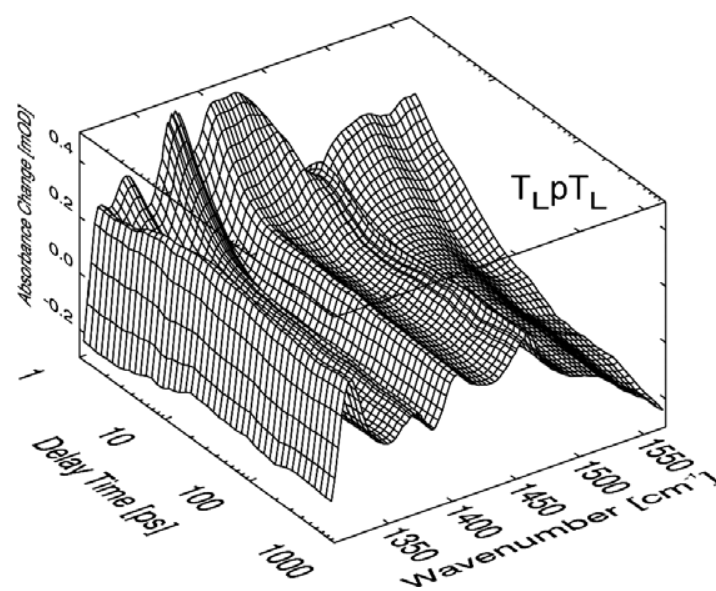

(a)

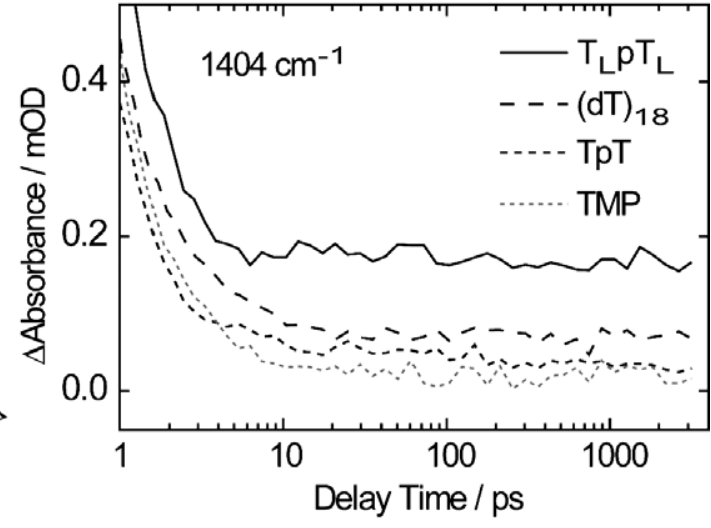

(b)

Fig. 2. (a) Transient absorption changes recorded for $\mathrm{T}_{\mathrm{L}} \mathrm{p} \mathrm{T}_{\mathrm{L}}$. (b) Comparison of the kinetic behavior for the different DNA model systems. Depicted is the time dependence of the absorption changes at the position of the CPD marker band at $1404 \mathrm{~cm}^{-1}$. 
be traced back to $\sim 1$ ps (see Fig. 2(a)). At very early delay times $<1$ ps (data not shown) the absorption signal contains additional contributions from non-linear coupling between pump and probe pulses, from higher excited states and from the IR absorption of the primarily excited state. These transients decay within the first picosecond. From transient UV/Vis experiments it is known that the monomer TMP exhibits an excited ${ }^{1} \pi \pi^{*}$ lifetime of the thymine base on the order of $700 \mathrm{fs}$ [6]. Therefore these results imply that for $\mathrm{T}_{\mathrm{L}} \mathrm{p}_{\mathrm{L}}$ the CPD lesion is formed (at least partially) during the decay of the initially excited ${ }^{1} \pi \pi^{*}$ state of thymine. Afterwards the corresponding absorption changes for CPD formation are constant over the entire time range.

Next we compare the results for the locked dinucleotide $\mathrm{T}_{\mathrm{L}} \mathrm{T}_{\mathrm{L}}$ with TMP, $\mathrm{TpT}$ and the oligomer (dT) $)_{18}$. In the dimerizable samples $\mathrm{TpT}$ and $(\mathrm{dT})_{18}$ we also find characteristic marker bands for CPD formation on the $1 \mathrm{ps}$ time scale. Looking at the time traces of the marker band at $1404 \mathrm{~cm}^{-1}$ in Fig. 2(b) we see a pronounced decay with less than 1 ps due to the decay of the ${ }^{1} \pi \pi^{*}$ state and the disappearance of time zero artifacts. Vibrational cooling manifests itself on a time scale of a few picoseconds. Thereafter the signals are essentially constant but differ in height for the four different samples. These constant absorption changes can be used as a measure for the "early" CPD yield $\phi_{\mathrm{CPD}}^{\mathrm{ps}}$ on the picosecond time scale. Their amplitudes show the following ordering: $\mathrm{TpT}<(\mathrm{dT})_{18}<\mathrm{T}_{\mathrm{L}} \mathrm{pT}$. This result is in line with the results from $\mathrm{CW}$ dimerisation yields $\phi_{\mathrm{CPD}}^{\mathrm{cW}}$ and gives evidence for a clear correlation of the two yields. In the following we present a quantitative analysis of the picosecond CPD-formation yield.

The excitation conditions in the time resolved experiment were identical for all samples. Thus the signal amplitudes can be directly analyzed as follows: TMP does not undergo photodimerisation. Therefore the observed absorption changes for this sample can be regarded as internal "zero line". The offset present in the traces of TMP at late delay times can be ascribed to heating of the solvent surroundings. This feature is due to the fact that most of the excitation energy is transformed into heat after the ultrafast ( $<1 \mathrm{ps)}$ internal conversion of $\sim 90 \%$ of the initially excited thymine bases. Intra- and inter-molecular vibrational cooling is most prominent before $10 \mathrm{ps}$. Later on a broad absorption change can be identified that is due to the heated $\mathrm{D}_{2} \mathrm{O}$ solvent (temperature change $\sim 50 \mathrm{mK}$ ) [23]. This contribution should be present for all samples and was subtracted during data analysis from the transient spectra of the other samples.

Assuming that CPD is the only photoproduct which contributes at the investigated wavenumber and taking into account the extinction coefficients for the different samples and the corresponding CPD lesions the picosecond quantum yield can be determined according to:

$$
\Delta A(\tilde{\nu})=\Delta \varepsilon(\tilde{\nu}) \cdot c^{*} \cdot d \cdot \varphi_{D}^{\mathrm{ps}}
$$

In Eq. (1) $\Delta \varepsilon(\tilde{\nu})$ represents the wavenumber dependent difference between the IR extinction coefficient of CPD $\varepsilon_{\mathrm{CPD}}(\tilde{\nu})$ and the one of the starting material $\varepsilon_{S}(\tilde{\nu}), \Delta \varepsilon(\tilde{\nu})=\varepsilon_{\mathrm{CPD}}(\tilde{\nu})-\varepsilon_{S}(\tilde{\nu}) . c^{*}$ is the concentration of the initially excited molecules and $d$ refers to the path length of the IR cell. Note that $c^{*}$ and $d$ are identical for all samples. Two approaches to compute the concentration $c^{*}$ were used. One is based on the parameters of the UV excitation pulse (its energy, its diameter at the sample location, and its absorption by sample), the other one on the magnitude of the initial bleach signal of the transient IR absorption with respect to the overall IR absorption. Either approaches yielded a concentration $c^{*}$ of $0.7 \pm 0.05 \mathrm{mM}$. The differences of the extinction coefficient $\Delta \varepsilon(\tilde{\nu})$ for the maximum of the most intense marker band at around $1320 \mathrm{~cm}^{-1}$ were obtained from IR spectra of the three dimerizable samples and their respective CPD forms. The extinction coefficients refer to one dimer and thereby two bases. The resulting values were $\sim 130 \mathrm{M}^{-1} \mathrm{~cm}^{-1}(\mathrm{TpT}), \sim 130 \mathrm{M}^{-1} \mathrm{~cm}^{-1}\left((\mathrm{dT})_{18}\right)$ and $\sim 200 \mathrm{M}^{-1} \mathrm{~cm}^{-1}\left(\mathrm{~T}_{\mathrm{L}} \mathrm{T}_{\mathrm{L}}\right)$. For 
the computation of the yields the absorption changes $\Delta A(\tilde{\nu})$ (corrected for TMP "zero-line") recorded after $\sim 100 \mathrm{ps}$ (averaging over three data points in respect to the delay time) were used. The yields $\phi_{\mathrm{CPD}}^{\mathrm{ps}}$ were determined to be $\sim 1.5 \%(\mathrm{TpT}), \sim 3 \%\left((\mathrm{dT})_{18}\right)$ and $\sim 10 \%\left(\mathrm{~T}_{\mathrm{L}} \mathrm{pT}_{\mathrm{L}}\right)$. This values match within the measurement uncertainty with the $\mathrm{CW}$ quantum yields $\phi_{\mathrm{CPD}}^{\mathrm{cw}}$ given above.

The values of the CPD-formation yield determined at $100 \mathrm{ps}$ combined with the constant amplitudes of the absorptions difference spectral in the marker bands observed at earlier times convincingly show that CPD formation is settled on the $1 \mathrm{ps}$ time scale. The hereby substantiated ultrafast formation of the CPD lesion was the prerequisite for the hypothesis that the (small) fraction of reactive conformers holds responsible for the (small) quantum yields observed. Indeed molecular dynamics simulations on thymidylyl $\left(3^{\prime}-5^{\prime}\right)$ thymidine (TpT) are in favor of the rareness of such reactive conformers [16]. They suggest that the CPD yield should scale with the thermal population of these reactive conformers. This is fully consistent with the more rapid saturation of CPD formation for poly(dT) or DNA from Escherichia coli in a rigid glass at $77 \mathrm{~K}$ as compared to room temperature aqueous solution [19,20]. At low temperatures reactive and non-reactive conformations are frozen in and may not interchange. Therefore CPD formation saturates when the limited number of reactive thymine bases are transformed. Whereas at room temperature thermal fluctuations cause a permanent interchange of the conformations. A test for this assumption is CD spectroscopy, which is a structural indicator for base stacking and allows evaluation of the amount of stacking in all thymine DNA. Previously Desnous et al. [7] have shown that TpT and $T_{L} p T_{L}$ differ in CD activity by a factor of 3.7 indicating that stacking of bases in $T_{L} p T_{L}$ is more efficient than in TpT. In our measurements (data not shown) we could confirm this result and found that the CD signal for $(d T)_{18}$ lies in between that of the two dinucleotides $T p T$ and $T_{L} p T_{L}$ [22]. The CD signatures of the three dimerizable samples thus have the same ordering as the picosecond quantum yields determined for CPD formation.

\section{Conclusion}

The presented results show that in $\mathrm{T}_{\mathrm{L}} \mathrm{pT}_{\mathrm{L}}$ and the other investigated dimerizable thymine model systems characteristic marker bands for CPD formation are present after $1 \mathrm{ps}$. There is no signal increase on the $100 \mathrm{ps}$ or ns time scale that could be related to further CPD formation. Additionally the determined picosecond quantum yields for CPD formation match the values reported from stationary experiments. This findings are consistent with an ultrafast $(2+2)$ cycloaddition via a singlet channel and rule out a triplet channel as a main path for CPD formation. The ultrafast nature of CPD formation explains the correlation between structural indicators and dimerisation yields. Thus giving evidence that the propensity for CPD damage is controlled by DNA structure. In the future it will be a main target to define the relative conformations of two neighboring thymine bases that represent reactive sites and are prone to CPD damage. Here further experiments on defined model systems and combined QM/MD calculations are expected to complete the picture of DNA photolesion formation.

\section{Acknowledgements}

This work was supported by the Deutsche Forschungsgemeinschaft through the DFG-Cluster of Excellence Munich-Centre for Advanced Photonics and SFB 749 (A5). We thank Prof. Bern Kohler for initiating some of the present research and Dr. Annett Bachmann for help in recording CD spectra. Dr. B. Ravindra Babu and Prof. Jesper Wengel are acknowledged for synthesis of the $T_{L} p T_{L}$ dimer. 


\section{References}

[1] M. Banyay, M. Sarkar and A. Graslund, Biophys. Chem. 104 (2003), 477-488.

[2] R. Beukers and W. Berends, Biochim. Biophys. Acta 41 (1960), 550-551.

[3] R. Beukers, A.P.M. Eker and P.H.M. Lohman, DNA Repair 7 (2008), 530-543.

[4] P.R. Callis, Annu. Rev. Phys. Chem. 34 (1983), 329-357.

[5] M.J. Cavaluzzi and P.N. Borer, Nucleic Acids Res. 32 (2004), e13.

[6] C.E. Crespo-Hernandez, B. Cohen and B. Kohler, Chem. Rev. 104 (2004), 1977-2019.

[7] C. Desnous, B.R. Babu, C. McIriou, J.U.O. Mayo, A. Favre, J. Wengel and P. Cliviot, J. Am. Chem. Soc. 130 (2008), 30-31.

[8] T. Douki, J. Photochem. Photobiol. B Biol. 82 (2006), 45-52.

[9] J. Eisinger and A.A. Lamola, Biochem. Biophys. Res. Commun. 28 (1967), 558-565.

[10] J.L. Hosszu and R.O. Rahn, Biochem. Biophys. Res. Commun. 29 (1967), 327-330.

[11] H.E. Johns, M. Delbruck and S.A. Rapaport, J. Mol. Biol. 4 (1962), 104-114.

[12] H.E. Johns, M.L. Pearson, C.W. Helleiner and J.C. Leblanc, J. Mol. Biol. 9 (1964), 503-524.

[13] G. Kallansrud and B. Ward, Anal. Biochem. 236 (1996), 134-138.

[14] M.K. Kuimova, J. Dyer, M.W. George, D.C. Grills, J.M. Kelly, P. Matousek, A.W. Parker, X.Z. Sun, M. Towrie and A.M. Whelan, Chem. Commun. 9 (2005), 1182-1184.

[15] W.M. Kwok, C. Ma and D.L. Phillips, J. Am. Chem. Soc. 130 (2008), 5131-5139.

[16] Y.K. Law, J. Azadi, C.E. Crespo-Hernandez, E. Olmon and B. Kohler, Biophys. J. 94 (2008), 3590-3600.

[17] S. Marguet and D. Markovitsi, J. Am. Chem. Soc. 127 (2005), 5780-5781.

[18] J.M. Martinez, S.K.C. Elmroth and L. Kloo, J. Am. Chem. Soc. 123 (2001), 12279-12289.

[19] R.O. Rahn and J.L. Hosszu, Photochem. Photobiol. 7 (1968), 637-642.

[20] R.O. Rahn and J.L. Hosszu, Photochem. Photobiol. 8 (1968), 53-63.

[21] T. Schrader, A. Sieg, F. Koller, W. Schreier, Q. An, W. Zinth and P. Gilch, Chem. Phys. Lett. 392 (2004), 358-364.

[22] W.J. Schreier, J. Kubon, N. Regner, K. Haiser, T.E. Schrader, W. Zinth, P. Clivio and P. Gilch, J. Am. Chem. Soc. 131 (2009), 5038-5039.

[23] W.J. Schreier, T.E. Schrader, F.O. Koller, P. Gilch, C.E. Crespo-Hernandez, V.N. Swaminathan, T. Carell, W. Zinth and B. Kohler, Science 315 (2007), 625-629.

[24] J.S. Taylor, Accounts Chem. Res. 27 (1994), 76-82.

[25] A.A. Vink and L. Roza, J. Photochem. Photobiol. B Biol. 65 (2001), 101-104.

[26] S.L. Zhang, K.H. Michaelian and G.R. Loppnow, J. Phys. Chem. A 102 (1998), 461-470. 


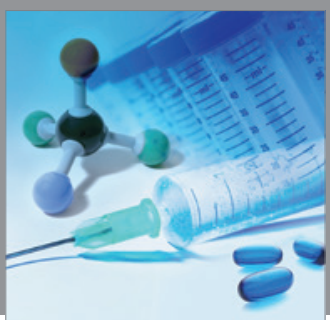

International Journal of

Medicinal Chemistry

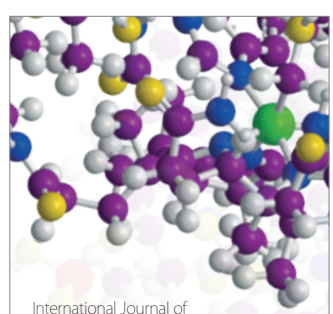

Carbohydrate Chemistry

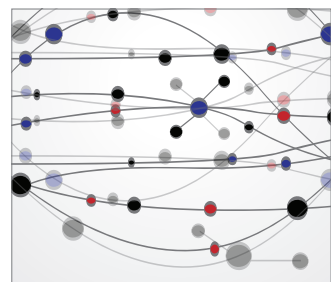

The Scientific World Journal
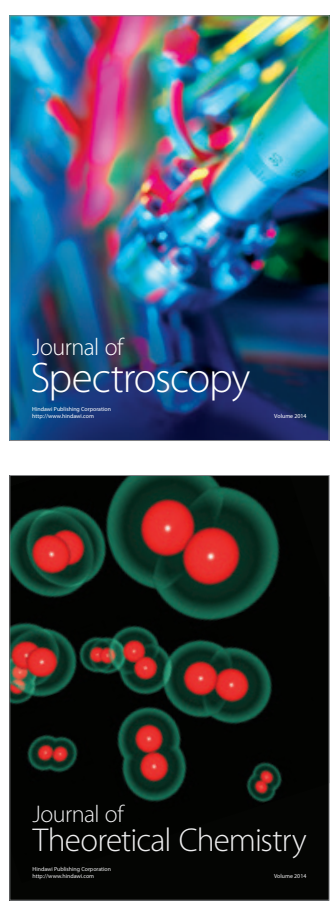
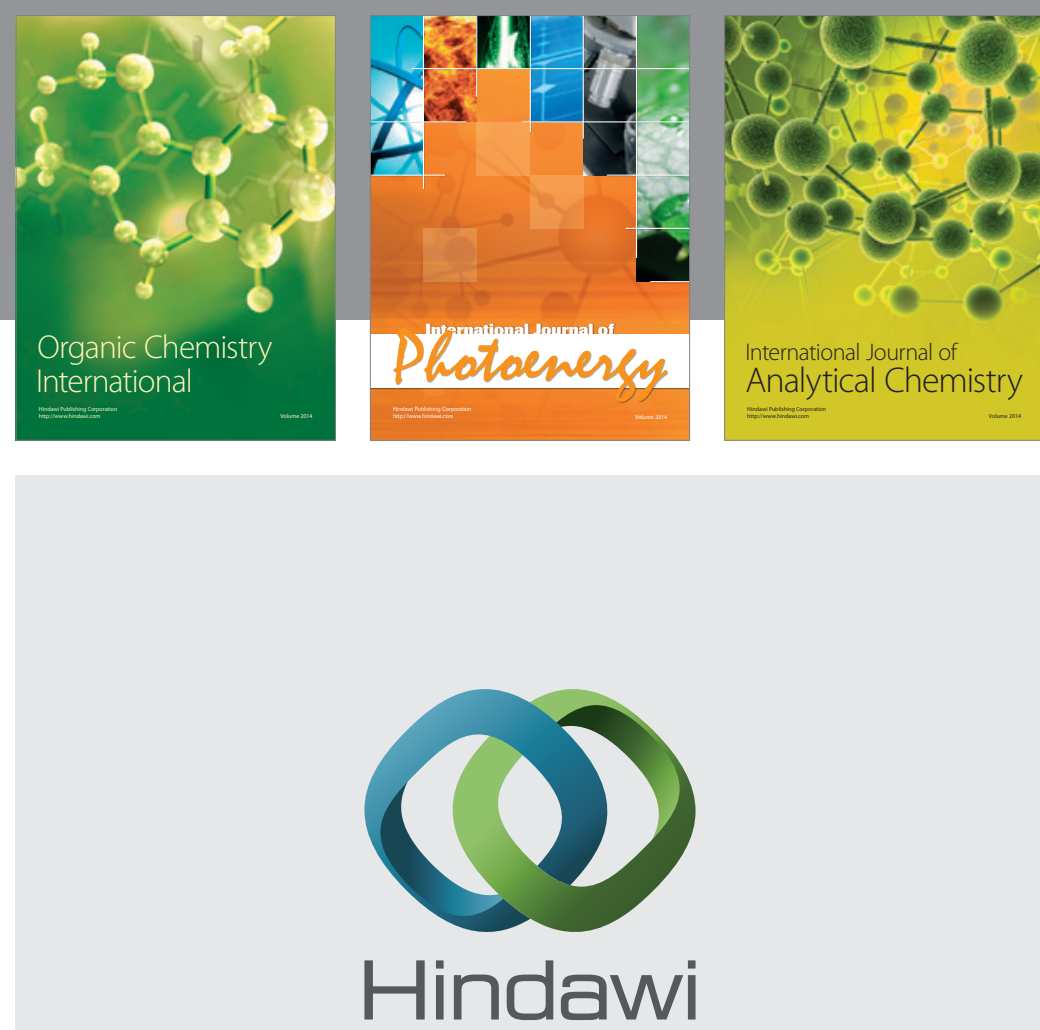

Submit your manuscripts at

http://www.hindawi.com
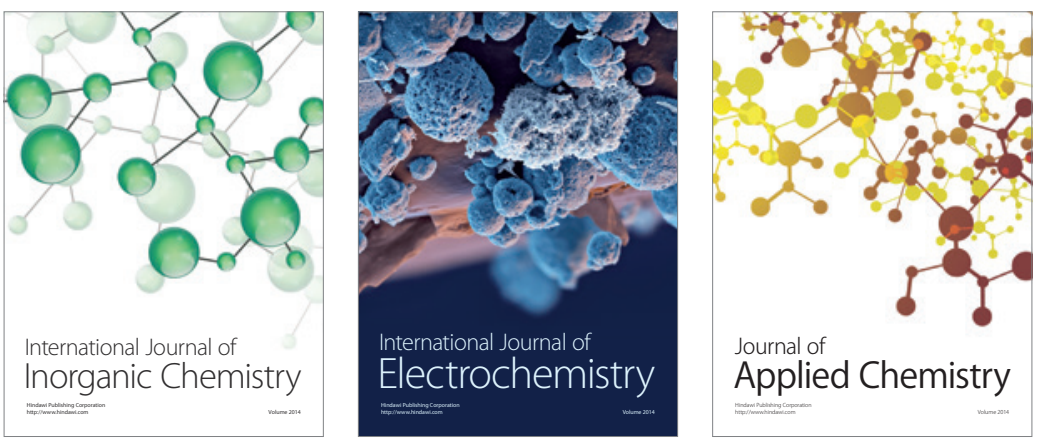

Journal of

Applied Chemistry
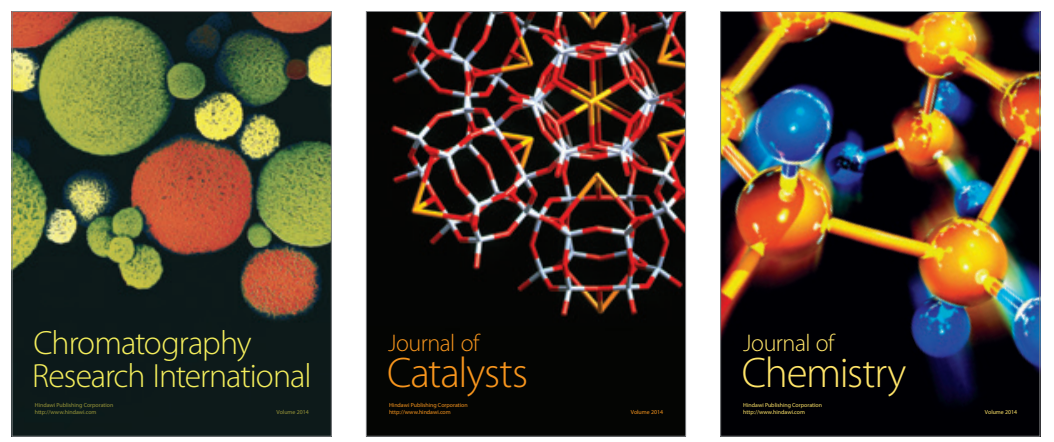
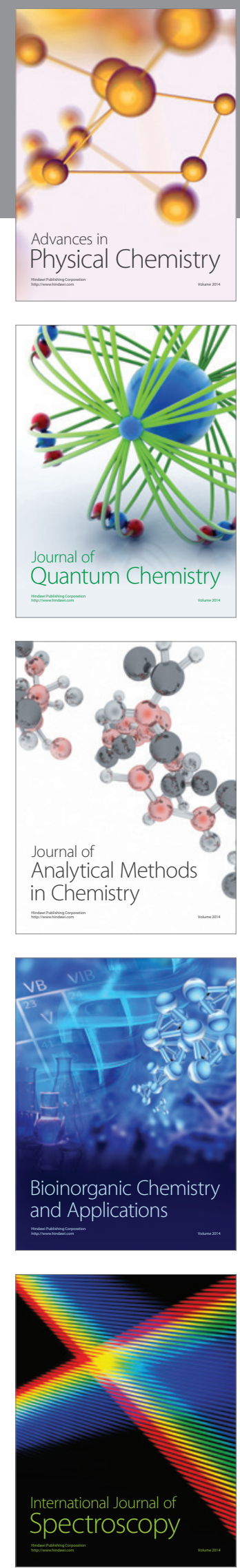